\title{
Applications of Bifurcation and Chaos on Discrete Time Dynamical System
}

\author{
Sharmin Akter \\ Lecturer \\ Department of Mathematics \\ Atish Dipankar University of \\ Science \& Technology \\ ,Dhaka,Bangladesh
}

\author{
Asia Khatun \\ Lecturer \\ Department of Mathematics \\ Atish Dipankar University of \\ Science \& Technology \\ Banani,Dhaka,Bangladesh
}

\begin{abstract}
In this paper the study of rigorous basic dynamical facts on bifurcation and chaos for discrete models in time dynamics and introduce a generalized logistic map and its dynamical behavior with tent and Henon Map has recognized.Different discrete curves have been developed and more general biological logistic curve are studied. Review and compare several such maps and analysis properties of those maps on the applications of bifurcation and chaos. Discuss the concept of chaos and bifurcations in the discrete time dynamical tent maps and generalized logistic growth models as time dynamical attractor.
\end{abstract}

\section{General Terms}

Fixed points, attracting points, repelling points, parameter, attractor, map, dynamical system

\section{Keywords}

Chaos, Bifurcations, Logistic Map, Tent Map, Henon Map, Periodic points, constant, chaotic behavior.

\section{INTRODUCTION}

The dynamical system concept is a mathematical formalization for any fixed "rule" which describes the time dependence of a point's position in its ambient space. The concept unifies very different types of such "rules" in mathematics: the different choices made for how time is measured and the special properties the ambient space may give an idea of the varsity of the class of objects described by this concept. There are different terms to describe the time dynamical system, such as, Chaos (roughly) as "a kind of order without periodicity." Wiggins says, "A dynamical system displaying sensitive dependence on initial conditions on a closed invariant set (which consists of more than one orbit) will be called chaotic. We often say observations are chaotic when there is no discernible regularity ororder." In particular, is generally characterized by,

Let $X$ be a compact metric space. A continuous map $f: X \rightarrow X$ is said to be chaotic on $X$ if $f$ satisfies the following properties:

(a) periodic points of $f$ are dense in $X$, (b) $f$ is topologically transitive, (c) $f$ has sensitive dependence on initial conditions,

Bifurcation, is about how dynamical systems change their behavior as some parameter of the system is changed. Otherwise A bifurcation is a qualitative change in an attractor structure as a control parameter. (for example,), we have to modify the differential equation to

$$
\frac{d P}{d t}=P(1-P)-H
$$

where $\mathrm{H}>0$ is the constant harvesting rate. Here is a simple example of a real-world problem modeled by a differential equation involving a parameter (the constant rate $\mathrm{H}$ ). Clearly, the fishermen will be happy if $\mathrm{H}$ is big, while ecologists will argue for a smaller $\mathrm{H}$ (in order to protect the fish population). First, let us look at the equilibria (or constant solutions) of this model. We must have

$$
p(1-p)-H-0
$$

which gives, $p=\frac{1 \pm \sqrt{1-4 H}}{2}$

1. if $\mathrm{H}<1 / 4$, then we have two constant solutions.

2. if $\mathrm{H}=1 / 4$, then we have one constant solution;

3. if $\mathrm{H}>1 / 4$, then we do not have constant solutions.

Therefore, $\mathrm{H}=1 / 4$ is a bifurcation value.

\section{PHYSICAL EXAMPLES OF CHAOS AND BIFURCATIONS}

1. Ignorance: this is labeled as "external influences". If you don't know what's happening than the result will be "surprising" and thus chaotic.

2. Many simple systems interacting in space: you know well how the single system behaves but the overall result can be surprising and thus chaotic. The simplest case is cellular automata. In this case interaction in space is essential to have chaos.

3. One single system developing in time: also, here if the law is recursive than the result (after some time) can be surprising and thus chaotic. Time in this case is essential to get chaos.

4. Use a quantum system like a radioactive material: the law is then intrinsically chaotic. There is no way to know when exactly a radioactive substance will decay. Such as population growth in the city, signaling system. 


\section{CHAOS AND BIFURCATION OF THE LOGISTIC MAP:}

The function $f_{a}(x)$ and $f_{a}^{n}(x)$ is a map of [0,1] into itself. Consider $f_{a}(x)=a x(1-x), \quad 0 \leq a \leq 4$.

Here, $x=0, x=p_{a}:=1-\frac{1}{a}$, are fixed points of $f$, where $0 \leq p_{a}<1$.

Note that changing the parameter $a$ changes the rate at which the asymptotic values $a_{\infty}$ is reached.

Here $f_{a}^{\prime}(x)=a-2 a x$.

Therefore, $f_{a}(x)$ has only one fixed point $x=0$ iff $a \leq 1$. For small growth rate $a<1$.

(say $a=1 / 2$ ), the population always dies out no matter what the initial condition is. In this case $a>1, f_{a}$ has two fixed points $x=0$ and $x=(a-1) / a$. Here,

$\max f(x)=f(1 / 2)=\frac{a}{4}$.

So, $f:[0,1] \rightarrow[0,1]$ if and only if $a \leq 4$. Here $f_{a}^{\prime}(0)=a$ and $f_{a}^{\prime}\left(p_{a}\right)=2-a$. Hence 0 is repelling fixed point for $a>1$ and attracting fixed point for $0<a<1$. Also $p_{a}$ is attracting for $1<a<3$ and is repelling if $a>3$ or $a<1$.

Here, $p_{a}$ is non-hyperbolic or neutral fixed point when $a=3$ is non-hyperbolic or neutral fixed point when $a=3$.

For periodic points with prime period 2, we have to consider $f^{2}(x)=x$.

Here, $a^{2} x^{2}-a(a+1) x+a+1$ has a real solution if and only if $a<-1$ or $a \geq 3$.

Thus $a^{2} x^{2}-a(a+1) x+a+1=0$ has the only one solution $x=2 / 3$.

For $a>3, a^{2} x^{2}-a(a+1) x+a+1=0$ has two real solution say $q_{1,2}$ where

$q_{1,2}=\frac{a+1 \pm \sqrt{(a+1)(a-3)}}{2 a}$.

We have $f\left(q_{1}\right)=q_{2}$ and $f\left(q_{2}\right)=q_{1}$, that is, $q_{1}, q_{2}$ are periodic points with prime period 2 for $a>3$.
Thus $q_{1}, q_{2}$ are attracting periodic points with prime period 2 if and only if $\left|\left(f_{a}^{2}\right)^{\prime}\left(q_{1}\right)\right|=\left|4+2 a-a^{2}\right|<1$, that is, $3<a<1+\sqrt{6} \approx 3.45$.

For $1+\sqrt{6}<a<3, q_{1}, q_{2}$ become unstable fixed point of $f^{2}(x)$. The 2 cycle disappears and is replaced by a 4 cycle, that is, four attracting periodic points with period 4.For $a \geq 1+2 \sqrt{2}$, there exists periodic points with prime period 3.

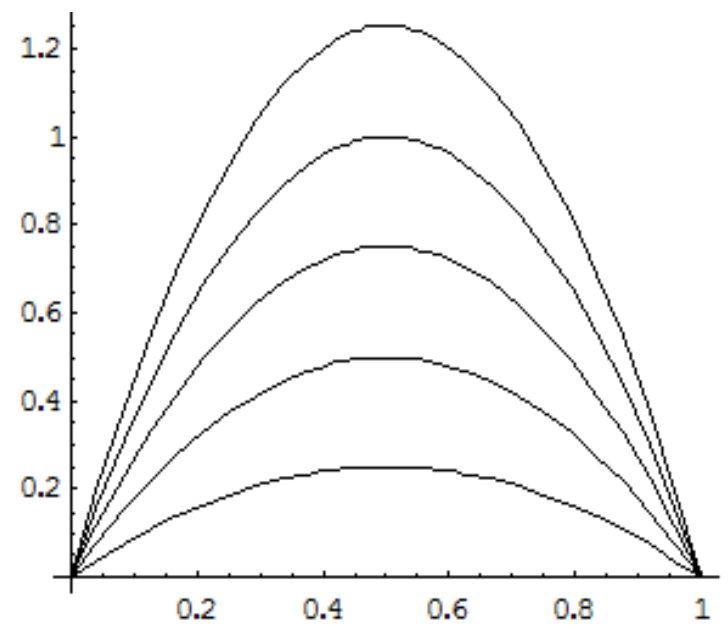

Figure 3.1: Logistic Map for different values of ' $a$ '

In the interval $3.45<a \leq 4$ changes occur rapidly, there is a three orbit when $a>3.6$. For $a=4$, the map is chaotic on $[0,1]$. For $a>2+\sqrt{5}$, there is a Cantor set in $[0,1]$ on which $f_{a}$ is chaotic when $a>2+\sqrt{5}$. Slight changes in the parameter, "a", of the function can cause the iterated function to change from stable and predictable behavior to unpredictable behavior which is called chaos. The behavior of the logistic function varies greatly as the parameter "a" change. The Following Figure illustrates some of the changes in this behavior.
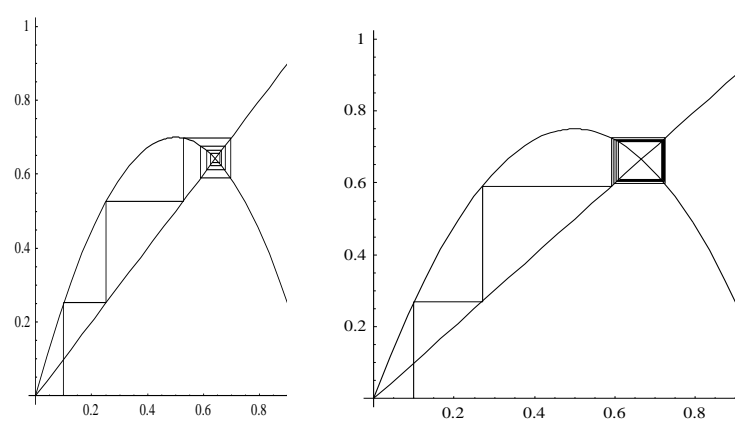

Figure 3.2: $\mathrm{a}=\mathbf{2 . 8 , 3}$ and Seed $=0.1$ 

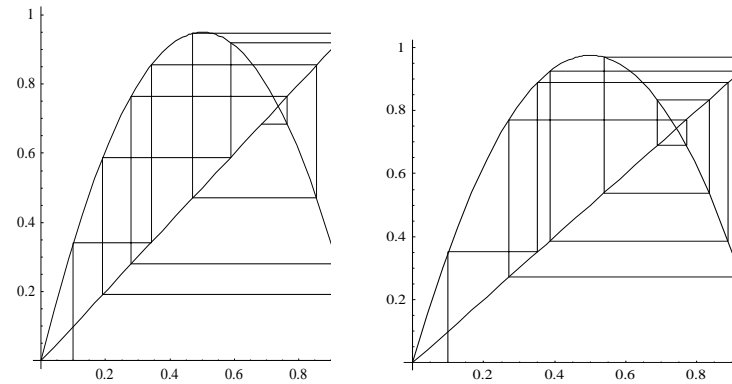

Figure 3.3: $\mathrm{a}=3.8,3.9$ and Seed $=0.1$

The fixed points for each of the above figures are:

In figure 2.2 , the graphical iteration converges on the fixed point.Thus, the fixed point is an attractor.

In figure 2.3, the graphical iteration appears to approach the fixedpoint but then begins to move away from it in a predictable patternover time. Thus, 0.667 is a repelling point.

If $\mathrm{a}=4$ in this logistic function, the iteration pattern behaviors in amuch different way. Figure given below shows this behavior for aseed $=0.2$ for a different number is iterations .
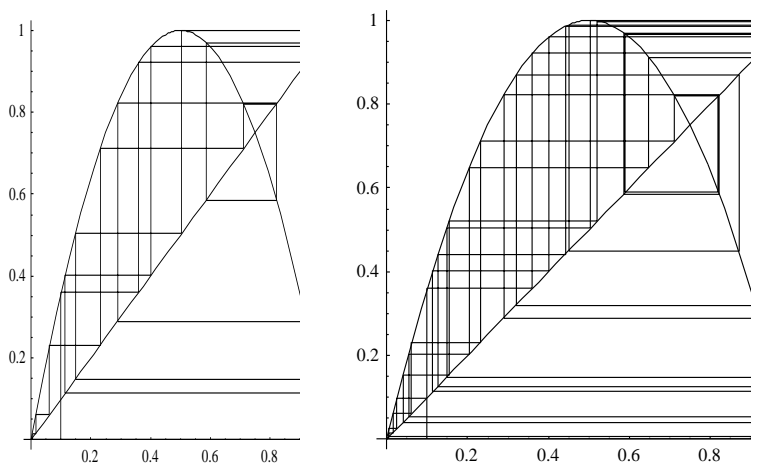

Figure 3.4: $a=4,20$ iterationsFigure 3.5: $a=4,40$ iterations

The fixed pointis $3 / 4$ the behavior of the iteration never seems to converge on any onepoint or pattern. Using many more iterations,theinterval $0<\mathrm{x}<1$ is filled. This behavior is very unstable.

For a $>3$ changes to repelling and a 2 cycle is born.

The system exhibits some interesting phenomena, which cannot be observed from the continuous logistic system.

For $3<a<1+\sqrt{6} \approx 3.45$, the two cycle is stable the population may oscillate between two values forever.

For $a=1+\sqrt{6} \approx 3.544090$, the 2 cycle becomes unstable and a stable 4 cycle is born then the population may oscillate between four values forever.

For $1+\sqrt{6}<a<1+2 \sqrt{2} \approx 3.544090$, the 4 cycle becomes unstable and a stable 8 cycle is born, when a is slightly bigger than 3.54 then 8 than 16,32 etc.

In general, a stable $2^{k}$ cycle is born at $a_{k}$ and becomes unstable at $a_{k+1}$, where $a_{1}=3$

$a_{5}=3.568759 \ldots$

$a_{2}=3.449 \ldots$

$a_{6}=3.569692 \ldots$

$a_{3}=3.54409 \ldots a_{7}=3.5698 \ldots$

$a_{4}=3.5644 \ldots \quad a_{8}=3.569946 \ldots$.

It should be clear that as $\mathrm{n}$ goes large these values are approaching a limit

$a_{\infty}=\lim _{n \rightarrow \infty} a_{n}=3.569946 \ldots$.

It means that an $\infty$ cycle for the value of a. Note that the successive bifurcations come faster and faster. The convergence is essentially geometric; the limit of large $n$, the distance between successive transitions shrinks by a constant factor

$\delta=\lim _{n \rightarrow \infty} \frac{a_{n}-a_{n-1}}{a_{n+1}-a_{n}}=4.6692016091 .$.

The number is called Feigenbaum constant.

In the interval $3.44<a \leq 4$ changes rapidly, there is a three on increasing a from 3 to 4 , periodic orbits arise at each step a doubling with the period.

It should be noted that $a_{\infty}<4$, which means that all iterates are confined. When $a>a_{\infty}$, behavior turns out to be chaos. As a increases, the fixed point $p_{a}$ becomes unstable for $a>3$.

For $3<a<a_{c}=3.569956 \ldots$, the system exhibits some interesting phenomena which cannot be observed from the continuous logistic system, when $a=3.9$ shown in the cobweb diagram.

For $a_{c}<a<4$. It is difficult to analysis this case. The sequence $\left\{x_{n}\right\}$ never settles down to a fixed point or a periodic point, instead the long term behavior is aperiodic.

For $\mathrm{a}=4$, the map is chaotic. The concept: An N-point attractor.

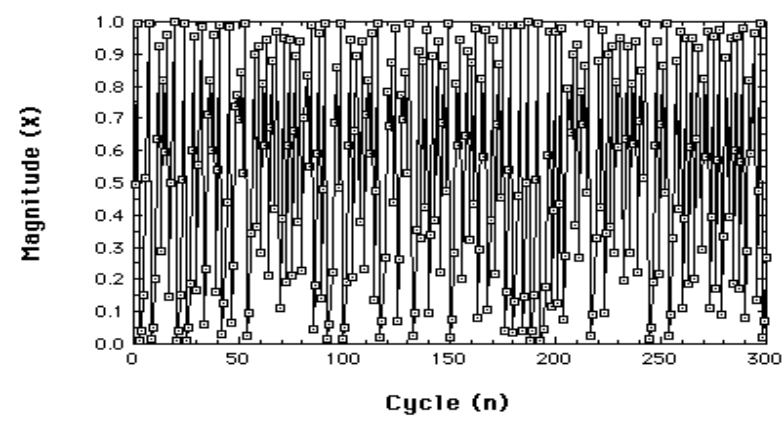

Figure 3.6: Chaotic behavior of the Logistic map at a= 3.99

Remark: The map needs to be redefined for a $>4$ because values $\mathrm{x}>1$ of can occur which ultimately divergent. 
Also, slope outside $0 \leq x \leq 1$ has magnitude strictly greater than 1 .

So, we restrict the control parameter a to the range

$0 \leq a \leq 4$. So that the logistic map maps the interval $0 \leq x \leq 1$ into itself. The behavior is much less interesting for other values of $\mathrm{x}$ and $\mathrm{a}$.

The logistic map $f_{a}(x)$ for different values of a.

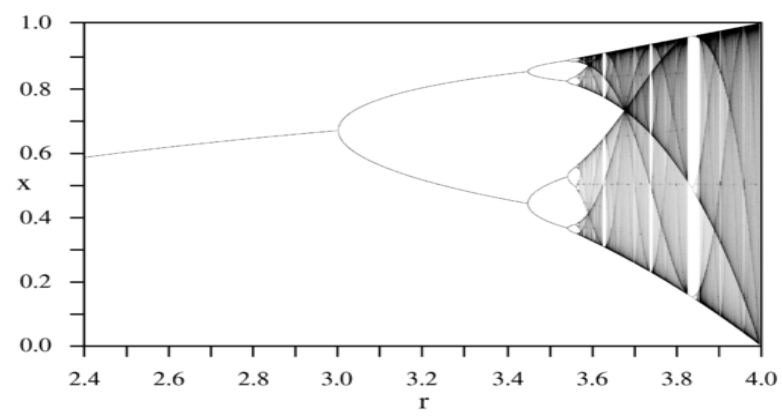

Figure 3.7: Bifurcation Diagram of Logistic Map

\section{CHAOS AND BIFURCATION OF THE TENT MAP}

Tent map is a conjugate, continuous, one-to-one and onto map. The chaotic behavior has given in below. By this

theorem we can discuss it accurately.

Theorem: Tent Map $\tau: X \rightarrow X=[0,1]$ is a chaotic dynamical system. Proof: We show that the map $\tau$ satisfies chaotic three conditions: -Periodic points of $\tau$ are dense in X. $\tau$ is one sided topologically transitive. $\tau$ has sensitive dependence one initial condition.

1. Periodic points of $\tau$ are dense in $X$

For any $\mathrm{x} \in \mathrm{X}$ and $\mathrm{n} \in \mid, \operatorname{Pn}(\tau) \cup\left(\mathrm{x}^{\frac{1}{2^{n}}}, \mathrm{x}+\frac{1}{2^{n}}\right) \neq \varnothing$

Let $\mathrm{x} \in \mathrm{X}$ and an open set $\mathrm{U}$ be given. Then there exist $\in>0$ such that $\mathrm{U}(\mathrm{x}, \in) \subset \mathrm{U}$.If we assume $\in<1$, then there

exist $\mathrm{n} \in \mid$ such that $\left(\mathrm{x}-\frac{1}{2^{n}}, \mathrm{x}^{\frac{1}{2^{n}}}\right) \subset \mathrm{U}(\mathrm{x}, \in) \in \mathrm{U}$.

Moreover, there exist xn such that $\mathrm{xn} \in \operatorname{Pn}(\tau) \cup\left(x-\frac{1}{2^{n}}, \mathrm{x}+\right.$ $\frac{1}{2^{n}}$

it follows that $x n \in \operatorname{Pn}(\tau) \cup U$. That is $\operatorname{Pn}(\tau) \cup U \neq \varnothing$. Hence $\operatorname{Pn}(\tau)$ is dense in $X$.

2. $\tau$ is one sided topologically transitive

For any $\mathrm{x} \in \mathrm{X}$ and $\mathrm{n} \in \mathrm{I},\left[\frac{k}{2^{n+1}}, \frac{k+1}{2^{n+1}}\right] \subset\left(\mathrm{x}-{\frac{1}{2^{n}}}_{\mathrm{x}+} \frac{1}{2^{n}}\right)$; $\mathrm{k}=\{0,1, \ldots 2 \mathrm{n}+1-1\}$

Let $\mathrm{U}$ and $\mathrm{V}$ are two non-empty open set in $\mathrm{X}$. Then there exist $\mathrm{x} \in \mathrm{U}$ and $\in>0$ such that $\left(\mathrm{x}-\frac{1}{2^{n}}, \mathrm{x}+\frac{1}{2^{n}}\right) \subset \mathrm{U}(\mathrm{x}, \in) \subset \mathrm{U}$. Moreover, there exist $\mathrm{k}$

such that $\left[\frac{k}{2^{n+1}}, \frac{k+1}{2^{n+1}}\right] \subset \mathrm{U}(\mathrm{x}, \in) \subset \mathrm{U}$. We also have that

$\left.\left[\frac{k}{2^{n+1}}, \frac{k+1}{2^{n+1}}\right]\right)=[0,1] . \quad$ Thus, $[0,1]=\quad \tau n+1($ $\left[\frac{k}{2^{n+1}}, \frac{k+1}{2^{n+1}}\right]$

$\cup \mathrm{V} \neq \varnothing$.

Hence $\tau$ is one sided topologically transitive.

3. $\tau$ has sensitive dependence one initial conditions

Let $\mathrm{x} \in \mathrm{X}$ be given. Then there exists $\mathrm{n} \in \mid$ such that $\frac{1}{2^{n}}<\epsilon$.

We assume that $\mathrm{x} \in\left[\frac{k}{2^{n}}, \frac{k+1}{2^{n}}\right]$ for some $\mathrm{k}=\{0,1, \ldots 2 \mathrm{n}$ -

1). Then $\left.\operatorname{\tau n}\left(\frac{k}{2^{n}}, \frac{k+1}{2^{n}}\right]\right)=[0,1]$. There exists $\mathrm{y} \in$ $\left[\frac{k}{2^{n}}, \frac{k+1}{2^{n}}\right] \subset \mathrm{U}(\mathrm{x}, \in)$ and we have $\mathrm{d}(\tau \mathrm{n}(\mathrm{x}), \tau \mathrm{n}(\mathrm{y})) \geq \frac{1}{2}$ $=\delta$.

Thus $\tau$ has sensitive dependence one initial condition. (Proved)

In mathematics, the tent map is an iterated function, in the shape of a tent, forming a discrete-timedynamical system.

It takes a point $x_{n}$ on the real line and maps it to another point:

$x_{n+1}=\left\{\begin{array}{cc}\mu x_{n} & \text { for } x_{n}<\frac{1}{2} \\ \mu\left(1-x_{n}\right) & \text { for } \frac{1}{2} \leq x_{n} .\end{array}\right.$

Where $\mu$ is a positive real constant.

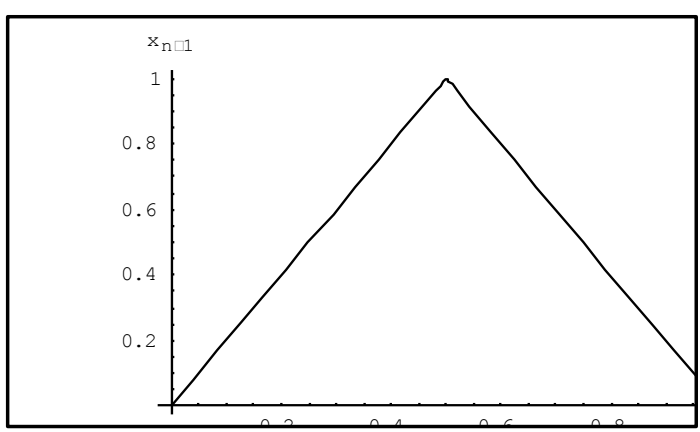

Figure 4.1: Graph of tent map function.

The tent map and the logistic map are topologically conjugate, and thus the behavior of the two maps are in this sense identical under iteration. 


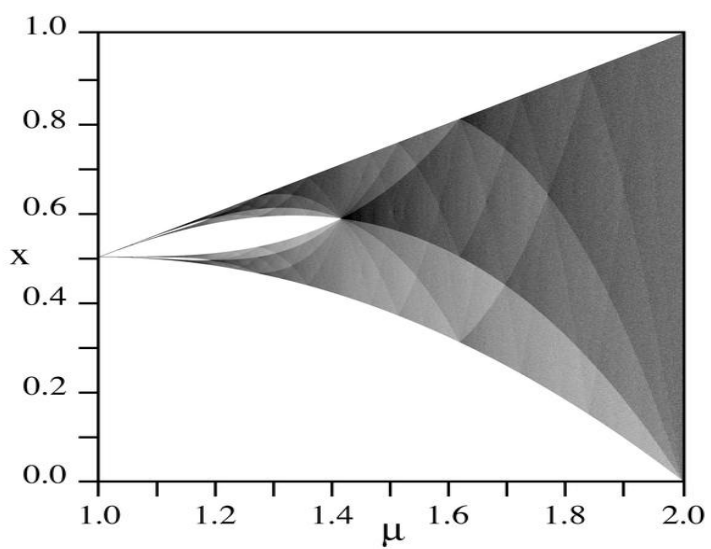

Figure 4.2: Bifurcation diagram for the tent map.

Higher density indicates increased probability of the $x$ variable

If $\mu$ is less than 1 the point $x=0$ is an attractivefixed point of the system for all initial values of $\mathrm{x}$ i.e. the systemwill converge towards $x=0$ from any initial value of $x$.

If $\mu$ is 1 all values of $x$ less than or equal to $1 / 2$ are fixed points of the system.

If $\mu$ is greater than 1 the system has two fixed points, one at 0 , and the other at $\mu /(\mu+1)$. Both fixed points are unstable i.e. a value of $\mathrm{x}$ close to either fixed point will move away from it, rather than towards it.

If $\mu$ is between 1 and the square root of 2 the system maps a set of intervals between $\mu-\mu 2 / 2$ and $\mu / 2$ to themselves. This set of intervals is the Julia set of the map i.e. it is the smallest invariant sub-set of the real line under this map. If $\mu$ is greater than the square root of 2 , these intervals merge, and the Julia set is the whole interval from $\mu-\mu 2 / 2$ to $\mu / 2$ (see bifurcation diagram).

If $\mu$ is between 1 and 2 the interval $[\mu-\mu 2 / 2, \mu / 2]$ contains both periodic and non-periodic points, although all of theorbits are unstable (i.e. nearby points move away from the orbits rather than towards them). Orbits with longer

lengths appear as $\mu$ increases.

\section{CHAOS AND BIFURCATION OF THE HENON MAP}

The Henon map is a discrete-time dynamical system. It is one of the most studied examples of dynamical systems that exhibit chaotic behavior. The Henon map is defined as

$x_{n+1}=y_{n}+1-a x_{n}^{2}$

$y_{n+1}=b x_{n}$

The map depends on two parameter as, $a$ and $b$, which for the canonical Henon map have values of $a=1.4$ and $b=0.3$ It is one of the most studied examples of dynamical systems that exhibit chaotic behavior. The Hénon map takes a point (x,y) in the plane and maps it to a new point $\mathrm{a}=1.4$ and $\mathrm{b}=0.3$

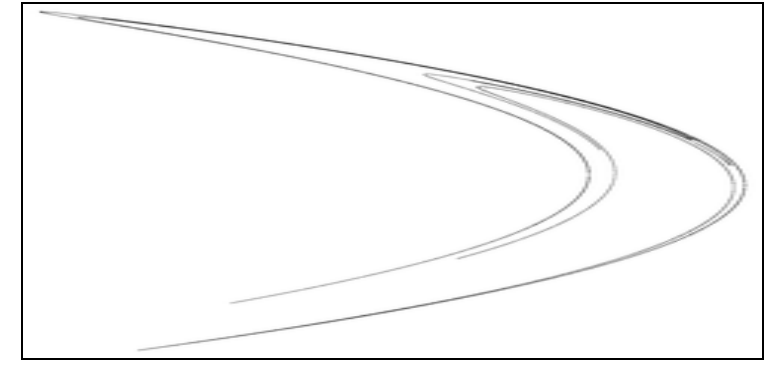

Figure 5.1: Hénon attractor for $a=1.4$ and $b=0.3$

The map depends on two parameters, $a$ and $b$, which for the canonical Hénon map have values of $a=1.4$ and $b=0.3$. For the canonical values the Hénon map is chaotic. For other values of $a$ and $b$ the map may be chaotic, intermittent, or converge to a periodic orbit. An overview of the type of behavior of the map at different parameter values may be obtained from its orbit diagram.

The Hénon maps two points into themselves: these are the invariant points. For the canonical values of $a$ and $b$ of the Hénon map, one of these points is on the attractor:

$$
\mathrm{x}=0.631354477 \ldots \text { and } \mathrm{y}=0.189406343 \ldots
$$

This point is unstable. Points close to this fixed point and along the slope 1.924 will approach the fixed point and points along the slope -0.156 will move away from the fixed point. These slopes arise from the linearization's of the stable manifold and unstable manifold of the fixed point. The unstable manifold of the fixed point in the attractor is contained in the strange attractor of the Hénon map.

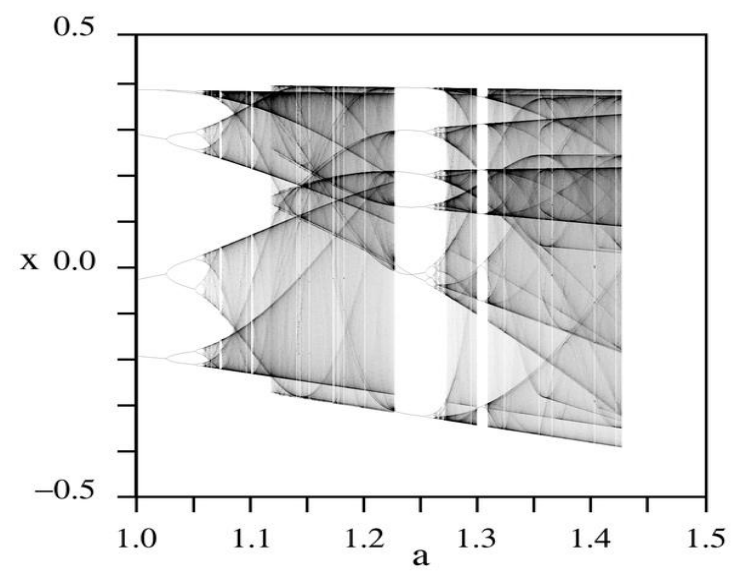

Figure 5.2: Bifurcation Diagram of Henon Map

The Hénon map does not have a strange attractor for all values of the parameters $a$ and $b$. For example, by keeping $b$ fixed at 0.3 the bifurcation diagram shows that for $\mathrm{a}=1.25$ the Hénon map has a stable periodic orbit as an attractor. Cvitanović and collaborators have shown how the structure of the Hénon strange attractor can be understood in terms of unstable periodic orbits within the attractor

\section{ACKNOWLEDGMENTS}

The beauty of the dynamics of the considered maps of the real line onto itself from the family becomes visible if we pass to the family $x_{n+1}$, where $\mathrm{n}$ is a complex variable and $\mathrm{c}$ is a complex parameter. The bifurcations among these maps becomes more efficient for Hopefully after reading this paper in its entirety, it will help you to get a better idea of how the dynamic system behaves as a whole. All things mentioned in 
this paper is to help you understand and describe discrete time dynamical system, for instance, it associated with a cycle of period $n$, where $n$ is large is hopefully a motivator to discover and learn different method what would more easily finding these points. It is by thoroughly grasping these concepts will you be able to go into the complexities of this topic.

\section{REFERENCES}

[1] Devaney, R.L., A First Course in Chaotic Dynamical Systems, Perseus Press, 1993.

[2] Devaney, R.L., an Introduction to Chaotic Dynamical Systems, Westview Press, 2003.

[3] Holmgren R.A, A First Course in Discrete Dynamical Systems, spring-verlag, 1996.
[4] Robert M. May, Simple Mathematical Models with very Complicated Dynamics, Nature 261, 459-467, 1976.

[5] M. Martelli, On the Definition of Chaos.

[6] Wiley-Interscience, Introduction to Discrete Dynamical Systems and Chaos, 1999.

[7] Li, T.-y., and Yorke J., Period Three Implies Chaos. American Mathematical Monthly 82 (1975), 985-992.

[8] L. Chuang, Rice University Lectures, http://math.rice.edu/ lukec/teaching/FA-05/MATH211/budworm.pdf 